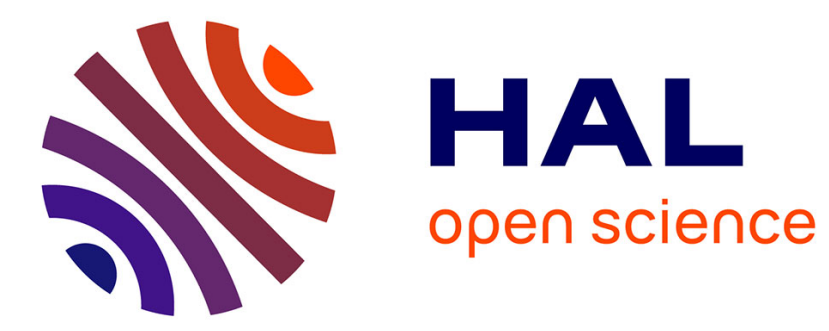

\title{
Les destins du virtuel à l'adolescence
}

Xanthie Vlachopoulou, Florian Houssier

\section{To cite this version:}

Xanthie Vlachopoulou, Florian Houssier. Les destins du virtuel à l'adolescence. Recherches en psychanalyse, 2013, 16 (2), 10.3917/rep.016.0178 . hal-01423234

\section{HAL Id: hal-01423234 \\ https://hal.science/hal-01423234}

Submitted on 7 Jan 2017

HAL is a multi-disciplinary open access archive for the deposit and dissemination of scientific research documents, whether they are published or not. The documents may come from teaching and research institutions in France or abroad, or from public or private research centers.
L'archive ouverte pluridisciplinaire HAL, est destinée au dépôt et à la diffusion de documents scientifiques de niveau recherche, publiés ou non, émanant des établissements d'enseignement et de recherche français ou étrangers, des laboratoires publics ou privés. 


\title{
LES DESTINS DU VIRTUEL À L'ADOLESCENCE
}

\author{
Xanthie Vlachopoulou et Florian Houssier
}

\section{Association Recherches en psychanalyse | Recherches en psychanalyse}

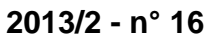

ISSN 1767-5448

Article disponible en ligne à l'adresse:

http://www.cairn.info/revue-recherches-en-psychanalyse-2013-2-page-178.htm

Pour citer cet article :

Vlachopoulou Xanthie et Houssier Florian, «Les destins du virtuel à l'adolescence », Recherches en psychanalyse, 2013/2 n¹6, p. 178-184.

Distribution électronique Cairn.info pour Association Recherches en psychanalyse.

(c) Association Recherches en psychanalyse. Tous droits réservés pour tous pays.

La reproduction ou représentation de cet article, notamment par photocopie, n'est autorisée que dans les limites des conditions générales d'utilisation du site ou, le cas échéant, des conditions générales de la licence souscrite par votre établissement. Toute autre reproduction ou représentation, en tout ou partie, sous quelque forme et de quelque manière que ce soit, est interdite sauf accord préalable et écrit de l'éditeur, en dehors des cas prévus par la législation en vigueur en France. II est précisé que son stockage dans une base de données est également interdit. 


\title{
Recherches en Psychanalyse - Research in Psychoanalysis
}

$16 \mid 2013$ - Psychanalyse et interdisciplinarité

Psychoanalysis and Interdisciplinarity

Images du virtuel

\section{Les destins du virtuel à l'adolescence}

The Destiny of the Virtual in Adolescence

[En ligne] 22 décembre 2013

\section{Résumé :}

Xanthie Vlachopoulou Florian Houssier

Comment expliquer l'attrait des adolescents ou des jeunes adultes pour les mondes numériques et, plus particulièrement, l'usage assidu des nouveaux modes de communication à distance rendus possibles par l'évolution des techniques?

Dans cet article, nous avons cherché, dans une perspective psychanalytique, à dégager des éléments de compréhension de l'investissement notable de ces nouvelles pratiques par les adolescents. Pour cela, nous revisitons les problématiques centrales du processus adolescent en les confrontant aux caractéristiques d'un jeu paradigmatique des mondes virtuels, les jeux de rôle en ligne. À partir de l'usage de ces jeux, notre hypothèse suggère que le recours privilégié aux mondes virtuels pourrait s'articuler avec le travail psychique propre au processus d'adolescence, notamment en termes d'étayage narcissique.

\section{Abstract:}

How might we explain the attraction of adolescents or of young adults to digital worlds and, particularly, the privileged use of new types of communication by distance that have been made possible by the evolution of technology?

In this paper we seek to explain the important investment of adolescents in these new practices from a psychoanalytical point of view. To this end, we review the central issues of the adolescent process by comparing them to the characteristics of a game that is a significant example of virtual worlds, the online role-playing games. Based on the use of these games, our hypothesis suggests that the privileged choice of the virtual world could be related to the psychological work which characterises the adolescence process, especially in terms of narcissistic shoring up.

Mots-clefs : mondes numériques, processus adolescent, travail psychique, virtuel, étayage narcissique

Keywords: digital worlds, adolescent process, psychological work, virtual, narcissistic shoring up

\author{
Plan : \\ Grandir en devenant des ascètes des mondes virtuels \\ Se construire en héros solitaire \\ Partir, connectés pour toujours \\ Devenir adulte ou rester dans le virtuel \\ Pour un travail du virtuel
}


Les techniques nouvelles de l'information et de la communication ont connu une expansion considérable et, pour un Français sur deux, le virtuel moderne fait partie intégrante de sa vie par l'usage quotidien d'un ordinateur (Deroin, 2010). Les adolescents et les jeunes adultes apparaissent comme ceux dont l'utilisation est la plus régulière, une grande majorité d'entre eux utilisant chaque jour ou presque les nouvelles techniques.

Comment expliquer cette appétence notable pour les mondes numériques? Pourquoi les adolescents qui naviguent dans ces espaces pixellisés se saisissent volontiers des nouveaux modes de communication où le distanciel triomphe sur le présentiel (Weissberg 1999) ?

Nous présentons cette rencontre entre adolescence et virtuel sous un angle avant tout clinique, à travers deux cas de jeunes joueurs excessifs de MMORPG $^{1}$, que nous avons rencontrés dans un cadre de recherche (Vlachopoulou, 2011) dont le protocole s'appuie sur trois entretiens cliniques semi-directifs et la passation d'épreuves projectives (Rorschach et TAT).

Notre choix des jeux de rôle en ligne, les MMORPG, a été déterminé, d'une part, par une constatation clinique, lui attribuant un statut potentiellement addictogène (Valleur 2002) et, d'autre part, par le fait que ces mondes virtuels présentent un condensé de pratiques diverses véhiculées par les nouvelles techniques.

Nous utilisons le terme virtuel non seulement dans le sens courant lié aux nouvelles techniques, mais également dans une perspective aristotélicienne : ce qui est potentiel, contenant les conditions de son actualisation. Pour les mondes des MMORPG, l'imprévisible des mises en situation s'accompagne de la malléabilité des issues possibles. Or, nul hasard que cette envergure du champ des possibles dans le jeu rencontre l'actualisation des potentialités des sujets adolescents confrontés à un avenir incertain et non déterminé. La virtualité du processus adolescent serait à entendre dans la dimension processuelle de cette traversée que constitue l'adolescence. Ce qui est virtuel durant le processus adolescent concerne la mise en suspens du potentiel d'adulte, en devenir; tout n'est pas prévu d'avance, ce processus et son issue ouvrent un imaginaire composé de multiples destins en suspens.

La littérature scientifique concernant ces mondes virtuels des MMORPG, nous a conformé dans notre choix d'un abord clinique psychanalytique de la question. En grande majorité, les recherches en psychologie clinique et psychopathologie abordent ces usages sous le prisme de la psychopathologie classificatoire (Hsu, Wen \& Wu, 2009; Collins, Freeman \& ChamarroRemuzic, 2011 ; Van Rooij, Schoenmakers, Vermulst, Van Den Eijnden \& Van De Mheen, 2011). L'approche clinique est très peu présente avec l'exception de quelques recherches ayant une perspective cognitivo-comportementale (Griffiths \& Meredith, 2009 ; Thorens, Khazaal \& Zullino, 2012). Ici, nous proposons une lecture clinique et psychopathologique d'orientation psychanalytique pour problématiser les effets de rencontre entre mondes virtuels et processus adolescent.

Au vu des mutations perpétuelles, le type de jeu virtuel que nous avons choisi pour cette recherche sera éphémère ; il n'en perd pas pour autant son caractère paradigmatique et nous servira de support commun pour étudier ces deux virtualités, adolescente et numérique.

Grandir en devenant des ascètes ${ }^{2}$ des mondes virtuels

Lucas est un adolescent de dix-neuf ans présentant une pratique excessive des MMORPG depuis de nombreuses années. Déscolarisé à l'âge de seize ans, il a connu un parcours chaotique, sur les plans professionnel comme personnel. Dans les nombreuses histoires qu'il nous a relatées, une grande difficulté à pouvoir contrôler sa pulsionnalité et tempérer ses réactions se dessinait, marquant une difficulté à canaliser l'intensité de mouvements pubertaires envahissants. Malgré une mobilisation défensive importante, notamment un recours au clivage, nous constatons des débordements 
possibles dans cet équilibre précaire. Le sentiment de persécution, très présent dans son discours, démontre une lutte contre des imagos parentales terrifiantes et des angoisses archaïques massives, ce que l'analyse des tests projectifs confirme. Ses assises narcissiques fragiles ne semblent pas pouvoir lui garantir suffisamment de sentiment de sécurité vis-à-vis de son monde interne. À travers les images pixellisées, il cherche une enveloppe contenante tout en maintenant une certaine distance avec les autres, la relation avec eux étant peut-être trop excitante et vécue comme menaçante. Ainsi, seul dans sa chambre, face à son écran, il semble éviter le contact physique grâce à cet espace virtuel, à la fois en prenant une distance avec ses parents, avec lesquels il partage de nouveau le même toit, mais également les autres, limitant le contact à des échanges par avatars interposés.

Théo, lui, est un adolescent de dix-huit ans qui s'est plongé dans les MMORPG à la suite de la mort de ses parents dans un incendie. La souplesse de son fonctionnement psychique lui permet d'élaborer ses conflits internes, mais, dans cette période fragilisante de deuil, il paraît avoir besoin d'un support mettant à distance la conflictualité, fantasmatiquement liée à une culpabilité associée à la mort de ses parents. Dans sa pratique des jeux MMORPG, il semblerait que Théo trouve le moyen de mettre en scène et d'exprimer une agressivité réprimée, car liée au niveau fantasmatique à la crainte d'avoir détruit l'objet. La confrontation aux autres est évitée, même à distance, Théo préférant se "déconnecter » quand la tension monte.

Ainsi, pour Lucas et Théo, dans des configurations différentes et à des degrés variables, une difficulté à gérer l'afflux pulsionnel pubertaire apparaît, et semble même conditionner en partie leur recours aux mondes virtuels. En effet comme nous précise R. Potier (2009), à partir d'une étude sur Second Life (SL), dans ces mondes virtuels :

[...] les frontières se brouillent et les corps s'effacent, [...] l'Autre existe dans l'interface de la communication, mais sans corps et sans visage, sans autre toucher que celui du clavier de l'ordinateur, sans autre regard que celui de l'écran. [...] Le corps y est absent, mais néanmoins sur-représenté par son absence ». (p. 152)

Les sensations corporelles liées à l'afflux pulsionnel pubertaire trouvent alors dans la décorporation liée au monde virtuel une issue garantissant un certain équilibre de leur fonctionnement psychique, en termes économiques. Dans une forme d'ascétisme moderne, ils semblent alors se protéger de cette charge pulsionnelle, qui les met en situation de crise. Dans leur discours, le rejet et la haine de la pulsion (Guillaumin, 2001) se manifestent à travers cette position phobique, rejoignant les observations initiales d'A. Freud (1936) à ce propos. Ce choix s'avère coûteux pour ces adolescents, dans le sens où leur isolement renvoie à un fantasme sacrificiel, ne serait-ce que par un mode de vie où le corps post-pubère est quasiment oublié, voire nié dans ses aspects pulsionnels les plus angoissants.

\section{Se construire en héros solitaire}

Chez Lucas, nous retrouvons des blessures narcissiques précoces, que l'adolescence vient réactiver; ces blessures contribuent à l'attraction exercée sur lui par les mondes virtuels, dans une quête de soutien narcissique non objectal. Confronté à une image négative de lui-même sous-tendue par des angoisses archaïques d'anéantissement présentes dans l'analyse de ses protocoles des tests projectifs (Rorschach et TAT), sa recherche de contenance l'amène à évoquer sa vie actuelle comme un moment difficile, caractérisé par le fait que « les choses le touchent énormément». Ce qu'il évoque comme étant des "crises de panique " semble traduire un moment d'impasse dans le processus adolescent avec un risque de décompensation accru. Ainsi, dans ce contexte, le recours à des relations médiatisées par ordinateur paraît plutôt témoigner du besoin de mise à distance de l'autre et de l'impossibilité $d^{\prime}$ 'envisager $d$ 'authentiques relations objectales. 
Pour Théo, nous n'avons pas repéré de défaillances majeures au niveau de la solidité de ses assises narcissiques, mais la difficulté d'aller vers la rencontre à l'autre et le besoin d'un étayage narcissique semblent déterminer son recours aux MMORPG. Dans les mondes virtuels, Théo montre tout de même son besoin de rencontres pour pallier sa solitude. Cherchant à être vu et se mettant au devant de la scène dans le jeu, il mobilise des défenses narcissiques majeures, en particulier le recours à l'idéalisation. Mais, audelà de cette image puissante et autosuffisante qu'il donne à voir, il semble plutôt se protéger de l'éventualité d'être à nouveau abandonné et délaissé, ce qu'implique la rencontre réelle avec l'autre.

Il ne s'agit peut-être pas tant d'une défaillance majeure qui conditionnerait ce recours au jeu virtuel, mais plutôt un besoin d'étayage narcissique ou objectal, qui se déclinerait de manière très variable selon les registres différents et les problématiques spécifiques.

Nos observations rejoignent ici les travaux sur la place du narcissisme dans cette pratique des jeux, soulignée par M. Stora (2004), mais que nous retrouvons également dans les travaux d'autres auteurs ayant étudié les jeux vidéo et les mondes virtuels. Au-delà de l'avatar, la valorisation et le soutien narcissique que peuvent offrir ces mondes, semble alors conditionner le recours à ces derniers qui prennent aussi la valeur équivalente d'une rêverie assistée et non d'un seul retrait narcissique, ligne de tension qui traverse l'usage des jeux vidéos (Houssier, Marty, 2010).

\section{Partir, connectés pour toujours}

Pour Lucas, le chemin vers l'élaboration de la perte semble difficile d'accès, le vécu de perte ne pouvant pas être reconnu; celle-ci le renvoie à sa propre mort, l'adolescence le confrontant à la nécessité de trouver qui il est tout en maintenant une certaine indifférenciation avec les objets perdus. L'absence n'est alors pas tant élaborée qu'associée au vide, sous-tendue par des angoisses d'anéantissement et de destruction.
Dans une représentation de soi mal différenciée de l'objet, il se trouve alors menacé de disparaître également. Dans sa pratique des MMORPG, une lutte contre ce vécu irreprésentable de la perte apparaît à plusieurs niveaux. En étant toujours " connecté ", il fait l'économie maniaque des deuils impossibles et de cette tristesse inaccessible car mise à distance dans cet espace virtuel où tout semble encore possible, sans conséquences définitives.

Pour Théo, cette question de la perte vient s'inscrire dans le contexte du décès tragique de ses parents. La confrontation à la perte brutale de ses premiers objets d'amour semble, alors, se traduire par une grande sensibilité à la perte dans cette période de deuil. Sans pour autant mettre en place des défenses massives, une tendance défensive anti-dépressive domine le tableau clinique. Le deuil de ses parents se trouve au premier plan, prenant la forme d'une quête de consolation et d'étayage qui transparaît dans ses récits. Dans les mondes virtuels, il semble alors trouver le soutien et l'étayage permanents d'un monde persistant, une sorte de retrouvaille avec la permanence des objets, immanents et par conséquent toujours à sa portée, à portée d'un " contact ". Dans ce monde virtuel, à l'abri de l'angoisse d'être à nouveau abandonné, il reste "connecté » aux autres et bénéficie de cette suspension du temps qui fait oublier la mort.

Ainsi, pour ces deux adolescents, le besoin de rester " connecté " prend un sens différent, mais semble tout de même révéler une difficulté certaine à élaborer les enjeux psychiques liés à la perte. En effet, rappelant le titre de l'ouvrage princeps dans l'étude du virtuel, La présence de I'absent $^{3}$, ces mondes paraissent particulièrement propices à pallier l'absence, la perte, la mort.

\section{Devenir adulte ou rester dans le virtuel}

Est-ce que le recours, notamment quand il s'avère excessif, aux mondes virtuels, serait lié à une tentative de maintenir la virtualité du processus adolescent face à la pression de 
l'actualisation du devenir adulte et les conflits intenses mobilisés?

Pour Lucas, la pratique des jeux de rôle en ligne était présente très tôt dans sa vie. Au départ, il s'agissait de jeux de rôle papier, puis assez rapidement une pratique sur Internet, toujours dans une certaine démesure, comme il l'indique. Dans l'histoire de sa pratique qu'il retrace, il semblerait, qu'au début, elle s'inscrivait dans une dimension ludique et socialisante, devenant un prétexte à la rencontre. En effet, il relate qu'à cette époque, les nombreuses rencontres $\mathrm{IRL}^{4}$ devenaient des grandes fêtes. Son usage actuel des jeux paraît cependant très éloigné de ces anciennes ambiances festives. Lorsqu'il fait part d'un mal-être accru, il nous donne l'impression d'une impasse du processus adolescent, sur fond de repli narcissique et d'angoisses profondes, posant la question d'une décompensation psychotique. Son recours excessif aux mondes virtuels semble s'inscrire d'une manière indirecte dans une lutte contre la pression pulsionnelle du processus adolescent, qui de par ses exigences internes de travail de subjectivation le fragilise et le mène dans une impasse inquiétante pour son intégrité identitaire.

Pour Théo, les enjeux de son recours aux mondes virtuels sont très différents de ceux qui conditionnent cette pratique excessive chez Lucas. En effet, malgré la souplesse de son fonctionnement psychique, pouvant lui donner les moyens d'élaborer les conflits de perte d'objet, Théo s'est trouvé confronté à la mort de ses parents. Ainsi, son recours aux MMORPG, s'est intensifié, et il semble passer de plus en plus de temps "connecté ». Théo cherche dans ces mondes virtuels un soutien face à ce deuil, dans une collusion entre mort réelle et enjeux de perte propres au processus adolescent.

Pour ces deux adolescents de vingt ans, même si une difficulté particulière à faire face aux pressions du devenir adulte se profile, nous ne pouvons pas attribuer leur recours aux mondes virtuels exclusivement aux bouleversements post-pubères sans penser aux aspects infantiles articulés au processus adolescent. Dans cette difficulté à élaborer la conflictualité adolescente, de multiples facteurs se superposent. En effet, ces données montrent l'intrication forte qui existe entre les exigences $d u$ processus adolescent et d'autres facteurs fragilisants, amenant dans une impasse face à laquelle les adolescents tentent de trouver une réponse par le recours aux mondes virtuels.

Le processus adolescent, lui-même, ne suffit pas à expliquer ce recours; $c^{\prime}$ est dans sa rencontre avec des problématiques empêchant le travail de l'adolescence amenant vers le devenir adulte que cette solution du virtuel est recherchée. En devenant ainsi des ascètes des mondes virtuels, face au grandir de la puberté et du pubertaire, en établissant des relations d'objet virtuel(les) (Missonnier 2007) face à l'imprévu de la rencontre avec l'autre, et en restant connectés pour mieux retarder le partir, signe d'une dépendance aux objets internes: c'est ainsi que le virtuel risque de figer la virtualité adolescente. Les rencontres avec les personnes en quête de ce virtuel ouvrent sur une autre interrogation.

\section{Pour un travail du virtuel}

Le recours au virtuel s'intègre-t-il dans le travail psychique propre au processus adolescent ou sert-il avant tout de modalité défensive antiélaborative?

Dans la réflexion freudienne sur les pulsions (Freud 1915), les destins pulsionnels se confondent souvent avec les défenses qui sont mises en place pour rétablir l'équilibre psychique en termes économiques, dans un appareil psychique débordé par l'intensité pulsionnelle. Mais la défense vient se distinguer d'un autre destin, celui du travail psychique qui intervient en temps de conflictualité exacerbée, porteur d'espoir d'évolution.

En revenant sur nos rencontres cliniques et en cherchant plus particulièrement ce qui a amené ces jeunes à se plonger dans les mondes virtuels des MMORPG, un dénominateur commun semble se dégager: le poids du devenir adulte en termes d'autonomisation et d'élaboration du passage de l'objet incestueux à l'objet extrafamilial. 
Nous ne pouvons apporter que des suggestions sur cette question, étant donné que nous avons rencontré ces adolescents au moment où le recours excessif aux jeux marquait un temps d'impasse subjective. Nous proposons cependant la possibilité de l'existence, d'une part, d'une opération du virtuel, et d'autre part, d'un travail du virtuel. Dans l'opération du virtuel, l'objet perdu sera remplacé par l'objet virtuel. D'une manière analogue, les images pixellisées viendront télescoper les images et les souvenirs de la réminiscence d'un travail de deuil psychique qui n'arrive pas à son cheminement. Les caractéristiques de l'objet seront attribuées aux images virtuelles. Le déni de la perte maintient, alors, le sujet dans une position de toute-puissance, lui évitant la souffrance; mais l'objet virtuel, restant externe et non intériorisé, annonce la répétition mortifère de l'addiction.
Le travail du virtuel serait d'un tout autre ordre. Le virtuel, ne venant pas se substituer à l'objet perdu, ouvre un espace qui permet d'approcher cet objet, car justement il n'a jamais vraiment été perdu : l'objet intériorisé est maintenu dans ses investissements grâce à la solidité des assises narcissiques.

Ainsi, face aux changements, à la perte, au deuil, le Moi va tenter de se consolider en investissant de nouveaux objets.

Les souvenirs de l'objet perdu jouent un rôle primordial dans la capacité du Moi à s'affermir ; le virtuel va alors éviter au sujet de se confronter à une perte et à un vacillement de son unité trop brutal.

L'investissement de l'objet virtuel, contribue au dégagement vis-à-vis de l'objet primaire, pour, progressivement, ouvrir sur des rencontres hors-virtuel.

\section{Bibliographie :}

Collins, E., Freeman, J. \& Chamarro-Premuzic, T. (2012). Personality traits associated with problematic and non problematic massively multiplayer online role playing game use. Personality and Individual Differences, 52, 133138.

Deroin, V. (2010). Diffusion et utilisation des TIC en France et en Europe en 2009. Culture chiffres.

Freud, A. (2009). Angoisse pulsionnelle à la puberté (1936). Le moi et les mécanismes de défense. Paris : PUF.

Freud, S. (1968). Pulsions et destin des pulsions (1915). Métapsychologie. Paris : Gallimard.

Griffiths, M. D. \& Meredith, A. (2009). Videogame addiction and its treatment. Journal of Contemporary Psychotherapy, 39, 247-253.

Guillaumin, J. (2001). Adolescence et désenchantement. Le Bouscat : L'esprit du temps.

Gutton, P. (2004). La virtualité et ses conduites. Adolescence, 22(1), 147-154.

Houssier, F. \& Marty, F. (2010). Adolescence, image et rêverie. Destins de la dépendance à l'objet. Cahiers de Psychologie Clinique, 35, 77-91.

Hsu, S., Wen, M. \& Wu, M. (2009). Exploring user experiences as predictors of MMORPG addiction. Computers \& Education, 53(3), 990-999.

Missonnier, S. (2007). Une relation d'objet virtuelle ? Le Carnet Psy, 120, 43-47.

Missonnier, S. \& Lisandre, H. (dir.) (2003). Le virtuel: la présence de l'absent. Paris : Éditions EDK.
Potier, R. (2009). Au risque du Virtuel ? Topique, 107(2), 149-162.

Stora, M. (2004). Addiction au virtuel. Le jeu vidéo. Adolescence, 22(1), 67-75.

Thorens, G., Khazaal, Y. \& Zullino, D. (2012). Traitement par thérapie cognitivo-comportementale d'une addiction Internet spécifique : un apport théorique illustré par une étude de cas d'un patient souffrant d'une addiction à un jeu de rôle en ligne massivement multijoueurs (MMORPG). Journal de Thérapie Comportementale et Cognitive, 22(2), 60-67.

Valleur, M. \& Matysiak, J.-C. (2002). Les addictions, Panorama clinique, modèles explicatifs, débat social et prise en charge. Paris : Armand Colin.

Van Rooij, A. J., Schoenmakers, T. M., Vermulst, A. A., Van Den Eijnden, R. J. J. M., \& Van De Mheen, D. (2011). Online video game addiction: Identification of addicted adolescent gamers. Addiction, 106, 205-212.

Vlachopoulou, X. (2011). Le virtuel et ses destins. Thèse de Doctorat. Soutenue le 7 octobre 2011. Université Paris Descartes.

Weissberg, J.-L. (1999). Présences à distance. Paris: L'Harmattan.

\section{Notes :}

${ }^{1}$ Ces initiales correspondent à l'appellation anglophone: "Massively Multiplayers On Line Role Playing Games », traduit en français par l'expression « Jeux de Rôle en Ligne Massivement Multi-joueurs ». 
${ }^{2}$ À partir de la réflexion de P. Gutton : "L'adolescent imagine les convenances de la fabrication du corps, leurs causes et leurs effets assurément interprétés comme externes. Ces conduites envahissantes et exigeantes en pensées et en actes peuvent chercher l'asymptote $d^{\prime}$ 'une virtualité pure [...] produisant alors un fantasme d'ascétisme » Gutton, P. (2004).
La virtualité et ses conduites. Adolescence, 22(1), 147-154.

${ }^{3}$ Missonnier, S. \& Lisandre, H. (dir.) (2003). Le virtuel : la présence de l'absent. Paris : Éditions EDK.

${ }^{4}$ IRL correspond à l'expression "In Real Life ", traduit en français comme "dans la vraie vie », c'est-à-dire en dehors du jeu.

\section{Les auteurs :}

\section{Xanthie Vlachopoulou}

Psychologue clinicienne, Docteur en Psychologie Clinique et Psychopathologie, membre associé du Laboratoire LPCP, Equipe E.A. 4056, Paris Sorbonne Cité.

\section{Florian Houssier}

Psychologue, Psychanalyste, Maître de conférences - HDR, Université Paris Descartes, Laboratoire LPCP, Equipe E.A. 4056, Paris Sorbonne Cité.

\section{Université Paris-Descartes}

71, avenue Édouard-Vaillant

92100 Boulogne-Billancourt

France

\section{Référence électronique}

Xanthie Vlachopoulou \& Florian Houssier, "Les destins du virtuel à l'adolescence ", Recherches en Psychanalyse [En ligne], 16|2013, mis en ligne le 22 décembre 2013.

Texte intégral

\section{Droits d'auteur}

Tous droits réservés 